\title{
OBTENÇÃO DO ÓLEO DA POLPA DE MACAÚBA (ACROCOMIA ACULEATA) A PARTIR DA EXTRAÇÃO COM LÍQUIDO PRESSURIZADO
}

\author{
C. P. TRENTINI ${ }^{1}$, L. CARDOZO-FILHO ${ }^{2}$ e C. SILVA $^{1}$ \\ ${ }^{1}$ Universidade Estadual de Maringá, Departamento de Tecnologia \\ ${ }^{2}$ Universidade Estadual de Maringá, Departamento de Engenharia Química \\ E-mail para contato: camiladasilva.eq@gmail.com
}

\begin{abstract}
RESUMO - O presente trabalho objetivou avaliar o desempenho de solventes orgânicos, em condições pressurizadas, na extração do óleo da polpa de macaúba. Os solventes utilizados na extração foram etanol e isopropanol. Os experimentos foram realizados em vazão fixa de solvente em $3 \mathrm{~mL} \mathrm{~min}^{-1}$ e pressão de 100 bar, avaliando o efeito da temperatura $\left(40\right.$ a $\left.80^{\circ} \mathrm{C}\right)$. $\mathrm{O}$ acréscimo da temperatura não ocasionou diferença significativa no rendimento em óleo $(\mathrm{p}>0,05)$ para a utilização do etanol como solvente, faixa de temperatura de 40 a $80{ }^{\circ} \mathrm{C}$. $\mathrm{Na}$ utilização do isopropanol como solvente, os rendimentos nas temperaturas de $40 \mathrm{e}$ $60{ }^{\circ} \mathrm{C}$ apresentam diferença significativa, no entanto, o aumento da temperatura de extração para $80{ }^{\circ} \mathrm{C}$ não ocasiona aumento do rendimento em óleo. Reporta-se que os melhores rendimentos obtidos com etanol e isopropanol, como fluidos pressurizados, não apresentam diferença significativa. Comparando as melhores condições de rendimento de óleo da ELP, etanol (43,9\%) e isopropanol (41,0\%), com o método convencional, o etanol apresentou $76,21 \%$ e o isopropanol $82,43 \%$ do rendimento obtido por Soxhlet, no entanto, em menor tempo de extração.
\end{abstract}

\section{INTRODUÇÃO}

A macaúba (Acrocomia aculeata) apresenta em sua composição óleo de alta qualidade, sendo considerada matriz oleaginosa com grande potencial para a produção sustentável de alimentos e biocombustíveis (Silva e Andrade, 2013). A macaúba apresenta melhores condições de rendimento em terras de cerrado, praticamente não sofre ataque de doenças e apresenta elevado teor de óleo ( $30 \%)$ (Abreu et al., 2011). O óleo da polpa possui uma coloração amareloalaranjado devido à presença de carotenóides e um acentuado grau de insaturação e apresenta elevado teor de ácido oleico (Hiane et al., 2005; Ramos et al. 2008).

Dentre os métodos convencionais para extração do óleo de sementes oleaginosas estão os métodos de prensagem e extração por solvente. O processo de extração por prensa mecânica realiza o esmagamento do material removendo parcialmente o óleo. Na extração de óleos vegetais por solventes normalmente se utiliza o Soxhlet e a extração líquida a quente, sendo que o solvente comumente utilizado é o $n$-hexano (Merck, 2006). Este método ocasiona a produção de resíduos indesejáveis, alto custo e tempo de processamento. Além do que, a alta temperatura para remoção do solvente remanescente no extrato, pode afetar a qualidade organoléptica do material (Regitanod'Arce, 2006). Com o crescimento da aplicação dos conceitos de química verde nos últimos anos (Prado, 2003; Sun et al., 2012), intensifica-se a busca por solventes e métodos alternativos para extração de óleos vegetais, os quais forneçam elevados rendimentos e resultem em um extrato com elevada qualidade e com a conservação dos compostos bioativos presentes na matriz . 
A extração com líquido pressurizado (ELP) é um processo que combina a temperatura e pressão, com solventes líquidos para alcançar a extração rápida e eficiente (Sagani et al., 2005). Uma grande vantagem da ELP em relação aos métodos de extração convencionais, é que os solventes sob pressão permanecem no estado líquido, mesmo acima dos seus pontos de ebulição a pressão atmosférica normal, o acréscimo da temperatura aumenta a solubilidade e a difusão dos analitos, diminuindo a viscosidade e a tensão superficial do solvente, fazendo com que ocorra uma maior penetração nos poros da matriz (Porschmann et al., 2001; Freitas et al., 2008). A ELP é utilizado para a extração de vários compostos em óleo vegetal como lipídios e ácidos graxos (Toschi et al., 2003), devido ao seu curto tempo de extração é também utilizado para extrações de compostos antioxidantes que se degradam durante longas extrações e temperaturas elevadas (Porschmann et al., 2001; Freitas et al., 2008).

Neste contexto, o presente trabalho objetiva avaliar a extração do óleo da polpa da macaúba utilizando etanol e isopropanol em condições pressurizadas, sendo obtida a cinética de extração em pressão fixa de 100 bar e temperaturas de 40 a $80{ }^{\circ} \mathrm{C}$, bem como a comparação dos rendimento com a extração por Soxhlet.

\section{MATERIAIS E MÉTODOS}

\subsection{Materiais}

Foram utilizados frutos de Macaúba da variedade Acrocomia aculeata, coletados na região do Cariri no estado do Ceará, Brasil. Para a extração por líquido pressurizado e por Soxhlet, foram utilizados acetato de etila $(99,5 \%)$, etanol $(99,8 \%)$ e isopropanol $(99,8 \%)$ de procedência JT Baker.

\subsection{Procedimentos Experimentais}

Preparo da matéria-prima: Os frutos da macaúba foram despolpados com o auxílio de faca inox sobre uma bandeja separando a casca, polpa, amêndoa e endocarpo. O material foi pesado, acondicionado em embalagens de plástico e armazenados em freezer a $-20^{\circ} \mathrm{C}$. As amostras foram mantidas congeladas até o momento da secagem. A polpa foi seca em estufa com circulação de ar a $60{ }^{\circ} \mathrm{C}$ por 8 horas. A amostra seca foi triturada em um moinho elétrico (IKA, modelo A 11/B) na velocidade de 14000 RPM e utilizou-se peneiras do tipo Tyler para a classificação granulométrica das amostras, com auxílio de um agitador mecânico de peneiras (Bertel, série 1.0), obtendo amostras com diâmetro médio de $0,5 \mathrm{~mm}$.

Extração por Soxhlet: A extração foi realizada conforme recomendado por Instituto Adolfo Lutz (2004). Foi utilizado tempo de extração de 480 minutos e 5 gramas de material. A temperatura de extração foi mantida constante acima da temperatura de refluxo do solvente, em todos os ensaios. Os ensaios foram realizados em duplicata. Após o tempo de extração o solvente remanescente no balão foi evaporado em estufa com circulação a $50{ }^{\circ} \mathrm{C}$ até peso constante.

Extração por Líquido Pressurizado: Os experimentos foram realizados com pressão e vazão constante de 100 bar e $3 \mathrm{~mL} \mathrm{~min}^{-1}$, respectivamente e com as temperaturas de 40,60 e $80{ }^{\circ} \mathrm{C}$. Utilizando uma célula extratora de aço inoxidável com capacidade para $15 \mathrm{~mL}$ e para pressurização do sistema uma bomba isocrática (Acuflow - série III). A célula foi preenchida com $6 \mathrm{~g}$ de amostra, aquecida e posteriormente preenchida com solvente, pressurizada e a amostra foi coleta em frascos âmbar. As amostras foram coletadas de 3 em 3 minutos e após 12 minutos de extração a cada 5 minutos, totalizando 72 minutos de extração, o solvente remanescente foi evaporado em estufa com circulação de ar a $50{ }^{\circ} \mathrm{C}$, até peso constante das amostras. $\mathrm{O}$ aparato experimental utilizado é reportado na Figura 1. 


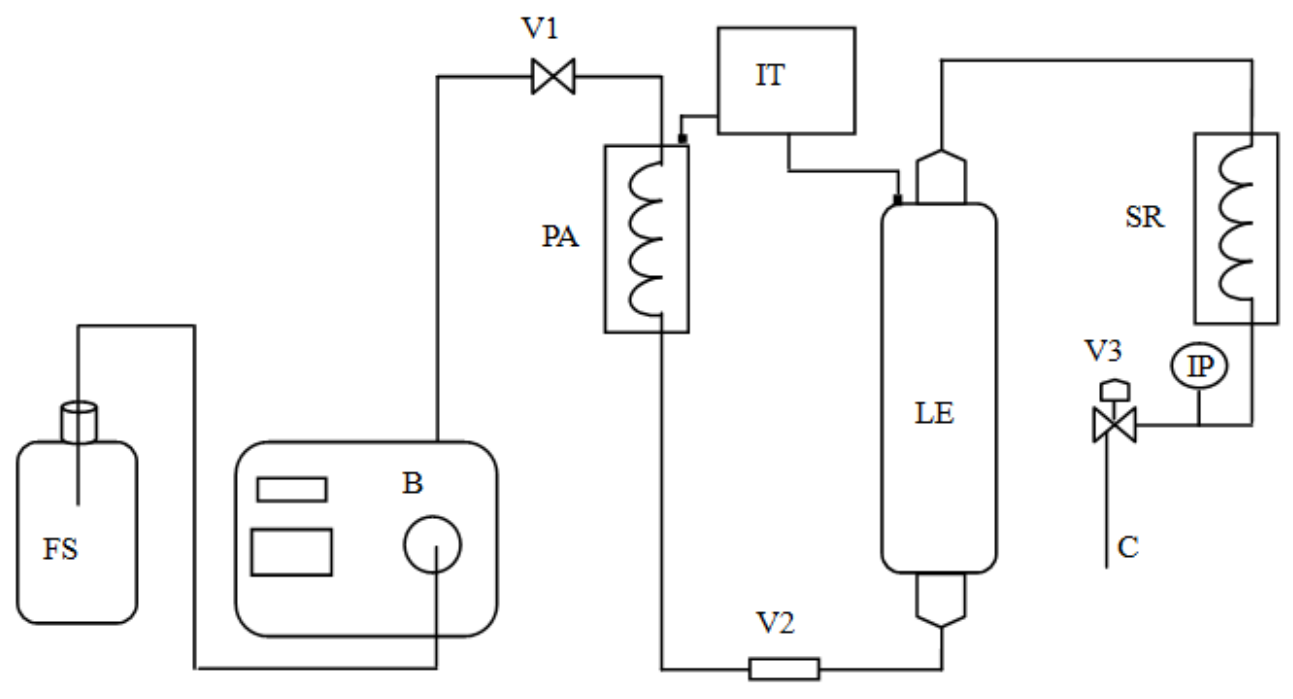

Figura 1 - Aparato Experimental utilizado na extração líquido pressurizado. (FS) Frasco de solvente; (B) Bomba isocrática; (V1) Válvula agulha; (PA) Pré-aquecimento; (V2) Válvula de retenção; (LE) Leito de extração; (IT) Indicador de temperatura; (SR) Sistema de resfriamento; (IP) Indicador de pressão; (V3) Válvula de controle de pressão; (C) Coleta do extrato.

\section{RESULTADOS E DISCUSSÕES}

A Tabela 1 apresenta as condições experimentais e rendimentos em óleo obtidos para as extrações por Soxhlet e ELP. Os resultados apresentados na Tabela 1 reportam que, para o etanol, a temperatura na faixa de 40 a $80^{\circ} \mathrm{C}$ não apresenta efeito significativo $(\mathrm{p}>0,05)$ no rendimento, no entanto, para o isopropanol este efeito é observado entre as temperaturas de 60 e $80^{\circ} \mathrm{C}$.

Moreau et al. (2003) reportam a extração do óleo de milho utilizando etanol e isopropanol como solventes, e os resultados obtidos pelos autores relatam que a extração com estes solventes apresentaram aumento do rendimento com o acréscimo da temperatura na faixa de 40 a $100{ }^{\circ} \mathrm{C}$, pressão fixa em 68,95 bar e 35 minutos de extração. Jaime et al. (2010) extraíram óleo de micro alga Haematococcus pluvialis utilizando etanol como solvente, onde a temperatura apresentou influencia positiva sobre o rendimento em óleo para os experimentos conduzidos em pressão fixa de 103,4 bar e 20 minutos de extração, obtendo 8,1 e 3,7\% de rendimento em óleo nas temperaturas de 50 e $100^{\circ} \mathrm{C}$, respectivamente.

Os resultados obtidos reportam que os maiores rendimentos em óleo, para ambos os solventes, não apresentam diferença significativa ( $\mathrm{p}>0,05)$. A extração do óleo pode ser atribuída à polaridade dos solventes, o etanol apresenta maior polaridade que o isopropanol (Takeuchi et al., 2009; Ramluckan et al., 2014). O etanol e o isopropanol por serem alcoóis podem demonstrar características bipolares, sendo adequados para extração de compostos bioativos (Almeida et al., 2012) e da fração lipídica (Rodriguez-Solana et al., 2014).

O elevado rendimento da extração por ELP foi reportado na literatura quando comparado com método de Soxhlet. Dunford e Zhang (2003) avaliaram o rendimento da extração do óleo do gérmen de trigo e reportam na temperatura de $105^{\circ} \mathrm{C}$, pressão de 103 bar e 30 minutos de extração rendimento de $\sim 23 \mathrm{e} \sim 17 \%$ utilizando etanol e isopropanol, respectivamente. Os autores relatam $10,8 \%$ de óleo utilizando o método de Soxhlet com $n$-hexano como solvente em 360 minutos de 
extração. Jesus et al. (2013) reportam a extração com condições pressurizas do óleo de palma utilizando o etanol como solvente, sendo que na temperatura de $60^{\circ} \mathrm{C}, 100$ bar e 60 minutos, obtiveram $68 \%$ de rendimento em óleo. Para a extração realizada por Soxhlet os autores reportam rendimentos de $61,3 \%$ em óleo com $n$-hexano como solvente e 360 minutos de extração.

Tabela 1 - Condições experimentais e rendimentos para as extrações com Soxhlet (SO) e com líquido pressurizado (ELP). Médias seguidas pela mesma letra minúscula (comparação entre as temperaturas do mesmo solvente) e letras maiúsculas (comparação dos melhores rendimentos entre os diferentes solventes na ELP) não se diferem estatisticamente $(p>0,05)$

\begin{tabular}{|c|c|c|c|c|c|c|}
\hline Solvente & $\begin{array}{l}\text { Tipo de } \\
\text { Extração }\end{array}$ & $\mathrm{T}\left({ }^{\circ} \mathrm{C}\right)$ & $\mathrm{P}$ (bar) & $\begin{array}{c}\text { Índice de } \\
\text { Polaridade }\end{array}$ & $\begin{array}{c}\text { Tempo } \\
(\mathrm{min})\end{array}$ & $\begin{array}{c}\text { Rendimento } \\
(\%)\end{array}$ \\
\hline \multirow{4}{*}{ Etanol } & \multirow{3}{*}{ ELP } & 40 & \multirow{3}{*}{100} & \multirow{3}{*}{5.2} & \multirow{3}{*}{72} & $41,89 \pm 2,17^{\mathrm{a}}$ \\
\hline & & 60 & & & & $43,48 \pm 3,47^{\mathrm{a}}$ \\
\hline & & 80 & & & & $43,78 \pm 1,55^{\mathrm{a}, \mathrm{A}}$ \\
\hline & SO & \pm 78 & - & 5.2 & 480 & $57,45 \pm 0,61$ \\
\hline \multirow{4}{*}{ Isopropanol } & \multirow{3}{*}{ ELP } & 40 & \multirow{3}{*}{100} & \multirow{3}{*}{3.9} & \multirow{3}{*}{72} & $30,43 \pm 0,97^{\mathrm{a}}$ \\
\hline & & 60 & & & & $37,77 \pm 0,32^{b}$ \\
\hline & & 80 & & & & $41,00 \pm 2,22^{\mathrm{b}, \mathrm{A}}$ \\
\hline & $\mathrm{SO}$ & \pm 82 & - & 3.9 & 480 & $47,86 \pm 0,68$ \\
\hline
\end{tabular}

Comparando as melhores condições de rendimento de óleo da ELP com o método convencional, o etanol apresentou $76,21 \%$ e o isopropanol $82,43 \%$ do rendimento obtido por Soxhlet, no entanto, em menor tempo de extração. Estes resultados evidenciam a característica da ELP que é o elevado rendimento em baixos tempos de extração.

As cinéticas de extração do óleo para com os solventes etanol e isopropanol são apresentadas nas Figuras 2 e 3, respectivamente. Os resultados apresentados na Figura 2 demonstram que a temperatura, na faixa avaliada, não apresenta influencia na taxa de extração em óleo para o etanol.

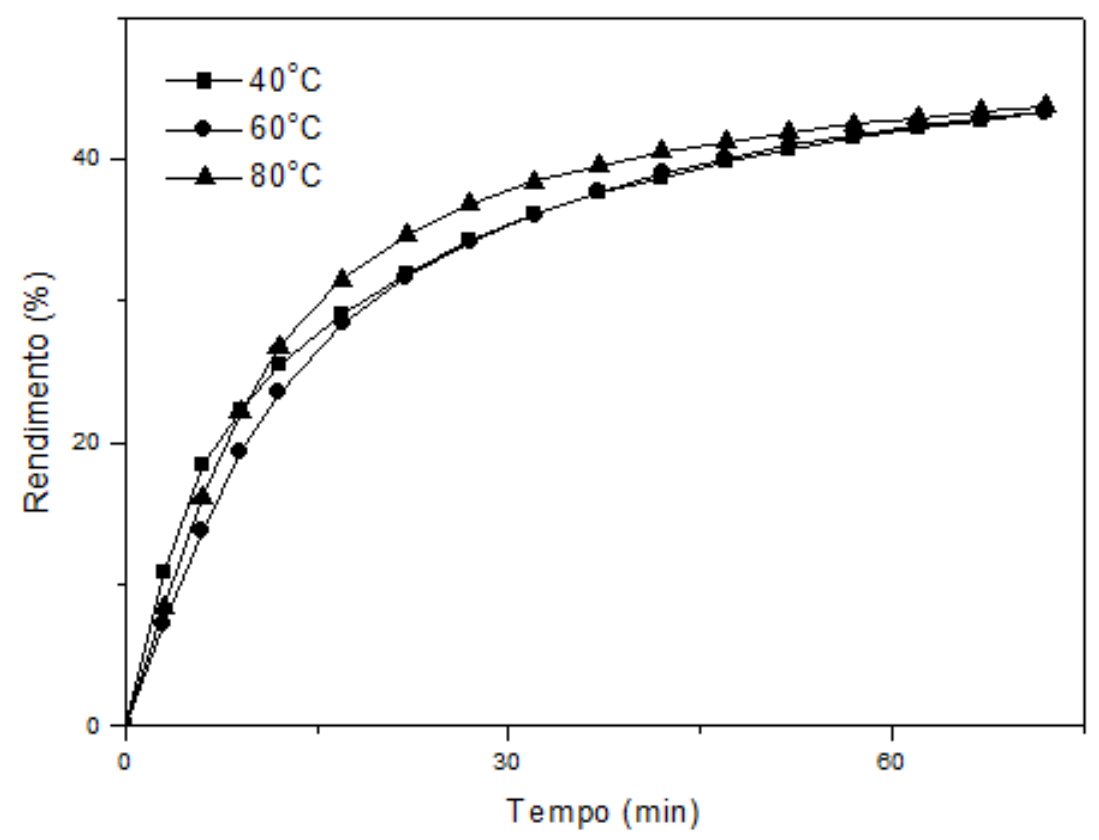


Figura 2 - Cinética de extração do óleo da polpa da macaúba com etanol.

$\mathrm{Na}$ extração com o isopropanol, conforme a Figura 3, o acréscimo da temperatura exerceu influência na extração do óleo da polpa da macaúba, a temperatura de $40{ }^{\circ} \mathrm{C}$ apresentou diferença significativa ( $>>0,05)$ no rendimento de óleo quando comparada com $60^{\circ} \mathrm{C}$, sendo que o aumento da temperatura para $80^{\circ} \mathrm{C}$ não apresentou diferença significativa $(\mathrm{p}>0,05)$ no rendimento de óleo a $60^{\circ} \mathrm{C}$.

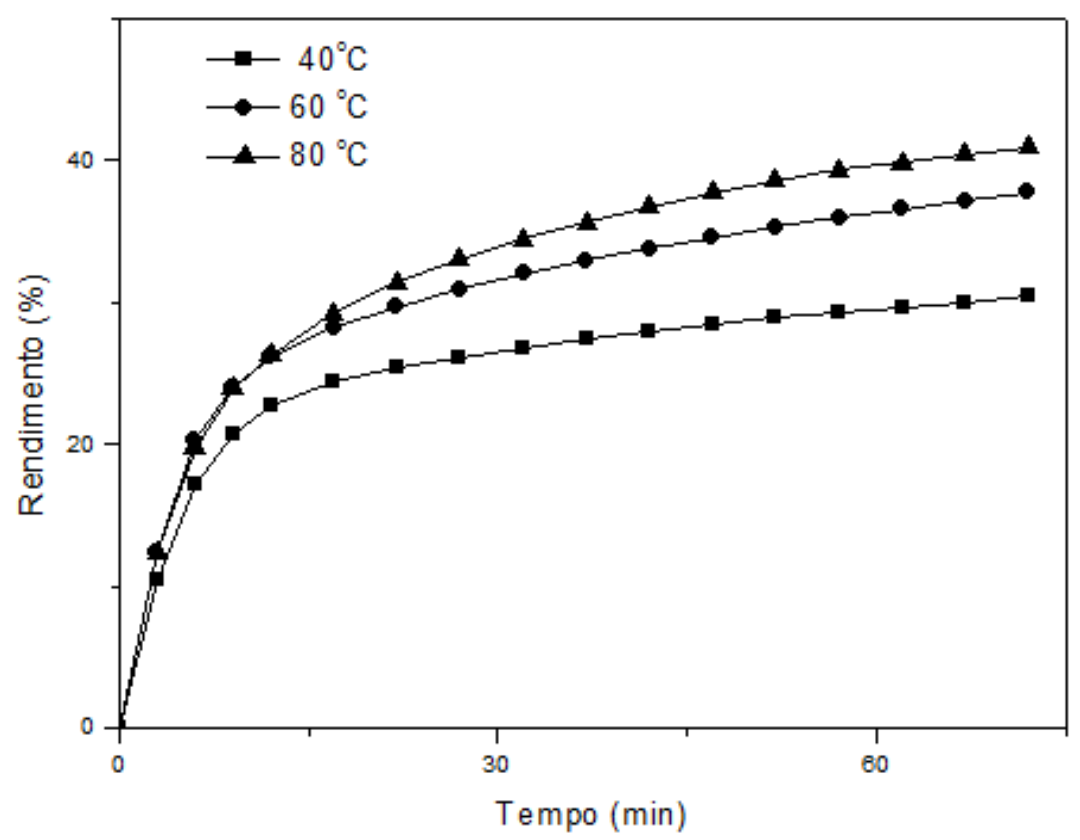

Figura 3 - Cinética da extração do óleo da polpa da macaúba com isopropanol.

\section{CONCLUSÃO}

Os resultados demonstraram o rendimento elevado da ELP do óleo da polpa da macaúba em baixos tempos de extração. Comparando as melhores condições de rendimento de óleo com o método convencional, o etanol apresentou 76,2\% e o isopropanol 82,4\% do rendimento obtido por Soxhlet, sendo que a extração por ELP apresenta menor tempo de extração. Os maiores rendimentos na ELP com etanol $(43,9 \%)$ e isopropanol $(41,0 \%)$ a $80{ }^{\circ} \mathrm{C}$ não apresentaram diferença significativa $(p>0,05)$. Nos rendimentos obtidos utilizando o etanol, as diferentes temperaturas não demonstraram diferença significativa $(\mathrm{p}>0,05)$ no rendimento em óleo. A extração utilizando o isopropanol à temperatura de $40{ }^{\circ} \mathrm{C}$ apresentou diferença significativa $(\mathrm{p}>0,05)$ em relação à temperatura de $60^{\circ} \mathrm{C}$, sendo que o aumento da temperatura para $80^{\circ} \mathrm{C}$ não exerceu diferença significativa $(\mathrm{p}>0,05)$ na extração.

\section{REFERENCIAS}

ABREU, I. S.; CARVALHO, C. R.; CARVALHO, G. M.; MOTOIKE, S. Y. First karyotype, DNA C-value and AT/GC base composition of macaw palm (Acrocomia aculeata) - a promising plant for biodiesel production. Australian J. Botan., v. 59, p. 149-155, 2011.

ALMEIDA, P. P.; MEZZOMO, N.; FERREIRA, S. R. S. Extraction of Mentha spicata L. 
Volatile Compounds: Evaluation of Process Parameters and Extract Composition. Food Bioproc. Technol. v. 5, p.548-559, 2012.

DUNFORD, N. T.; ZHANG, N. Pressurized solvent extraction of wheat germ oil. Food Research Internat., v. 36, p. 905-909, 2003.

FREITAS, L. S.; JACQUES, R. A.; RICHTER, M. F.; SILVA, A. L.; CARAMAO, E. B. Pressurized liquid extraction of vitamin E from Brazilian grape seed oil. J. Chromat. A, v. 1200, p.80-83, 2008.

HIANE, P. A., RAMOS FILHO, M. M., RAMOS, M. I. L., MACEDO, M. L. R. Bocaiúva, Acrocomia aculeata (Jacq.) Lodd., pulp and kernel oils: characterization and fatty acid composition. Br. J. Food Technol., v.8, p.256-259, 2005.

INSTITUTO ADOLFO LUTZ. Métodos Físico-Químicos para Análise de Alimentos - $4^{\mathrm{a}}$ Edição, $1^{\mathrm{a}}$ edição digital, 2004.

JAIME, L.; RODRIGUEZ-MEIZOSO, I.; CIFUENTES, A.; SANTOYO, S.; SUAREZ, S.; IBANEZ, E.; SENORANS, F.J. Pressurized liquids as an alternative process to antioxidant carotenoids' extraction from Haematococcus pluvialis microalgae. LWT Food Scienc. Technol., v. 43, p. 105-112, 2010.

JESUS, A. A., ALMEIDA, L. C., SILVA, E. A., EGUES, S., FRANCESCHI, E., FORTUNY, M., SANTOS, A. F., ARAUJO, J., SOUZA, E. M. B. D., DARIVA, C. Extraction of palm oil using propane, ethanol and its mixtures as compressed solvent. J. Supercrit. Fluids, v.81, p. 245-253, 2013.

MERCK. Ficha de informações de segurança de produtos químicos: $n$-hexano. São Paulo: Merck S. A. 2006.

MOREAU, R. A.; POWELL, M. J.; SINGH, V. Pressurized Liquid Extraction of Polar and Nonpolar Lipids in Corn and Oats with Hexane, Methylene Chloride, Isopropanol, and Ethanol. JAOCS, v. 80, n. 11, 2003.

REGITANO-D'ARCE, M. A. B. Extração e Refino de óleos vegetais. In: OETTERER, M.; REGITANO-D'ARCE, M. A. B.; SPOTO, M. H. F. Fundamentos de Ciência e Tecnologia de Alimentos. Manole, p. 300-351, 2006.

PORSCHMANNA, J.; PLUGGEA, J.; TOTH, R. In situ derivatisation using pressurized liquid extraction to determine phenols, sterols and carboxylic acids in environmental samples and microbial biomasses. J. Chromat. A, v. 909, p. 95-109, 2001.

PRADO, A. G. S. Química Verde, Os Desafios da Química do Novo Milênio. Quím. Nova, v.26, p.738-744, 2003.

RAMLUCKAN, K.; MOODLEY, K. G.; BUX, F. An evaluation of the efficacy of using selected solvents for the extraction of lipids from algal biomass by the soxhlet extraction method. Fuel, v. 116, p. 103-108, 2014.

RAMOS, M. I. L., RAMOS FILHO, M. M., HIANE, P. A., BRAGA NETO, J. A., SIQUEIRA, E. M. A. Qualidade nutricional da polpa de bocaiúva Acrocomia aculeata (Jacq.) Lodd. Ver. Ciênc. tecnol. Alim., v.28, p. 90-94, 2008. 
RODRÍGUEZ-SOLANA, R.; SALGADO, J. M.; DOMÍNGUEZ, J. M.; CORTÉS-DIÉGUEZ, S. Characterization of fennel extracts and quantification of estragole: Optimization and comparison of accelerated solvent extraction and Soxhlet techniques. Ind. Crops Prod., v. 52, p. 528- 536, 2014.

SANAGI, M. M.; SEE, H. H.; IBRAHIM, A. W.; NAIM, A. A. Determination of carotene, tocopherols and tocotrienols in residue oil from palm pressed fiber using pressurized liquid extraction-normal phase liquid chromatography. Analyt. Chim. Acta, v. 538, p. 71-76, 2005.

SILVA, G. C. R., ANDRADE, M. H. C. Development and simulation of a new oil extraction process from fruit of macauba palm tree. J. Food Process Eng., v.36, p.134-145, 2013.

TAKEUCHI, T. M; PEREIRA,C. G.; BRAGA, M. E. M.; MARÓSTICA,M. R. JR.; LEAL,P. F; MEIRELES, M. A. A. Low-Pressure Solvent Extraction (Solid-Liquid Extraction, Microwave Assisted, and Ultrasound Assisted) from Condimentary Plants. In: Meireles, M. A. A. Extracting bioactive compounds for food products: theory and applications. CRC Press, p. 137-218, 2009.

TOSCHI, A. T.; BENDINI, G.; RICCI, A.; LERCKER, G. Pressurized solvent extraction of total lipids in poultry meat. Food Chem., v. 83, p. 551-555, 2003.

SUN, H.; GE, X.; LV, Y.; WANG, A. Application of accelerated solvent extraction in the analysis of organic contaminants, bioactive and nutritional compounds in food and feed. J. Chromat., v.12, p. 1-23, 2012. 